\title{
Obstructive sleep apnea representations, self-efficacy and family coping regarding APAP adherence: a longitudinal study
}

\author{
Rute Sampaio $^{\mathrm{a} *}$, M. Graça Pereira ${ }^{\mathrm{a}}$ and João C. Winck ${ }^{\mathrm{b}}$ \\ ${ }^{a}$ School of Psychology, Minho University, Braga, Portugal; ${ }^{b}$ Department of Medicine, Oporto \\ University, Porto, Portugal
}

(Received 8 November 2012; final version received 5 February 2013)

\begin{abstract}
Adherence is still an important issue considering new advances in Obstructive Sleep Apnea (OSA) treatment, as automatic positive airway pressure (APAP). The aim of the present study was to identify and explore relationships between identified predictors of adherence, over time. After overnight sleep study and OSA diagnosis and during a six-month APAP treatment period, a total of 153 patients underwent a three time psychological protocol evaluation. Generalized estimating equations were applied to analyzed repeated measurements in the same individuals. Results show that $40 \%$ of patients were poorly adherent and $60 \%$ were adherent after six months of treatment. The results confirmed a predictive value of age, self-efficacy, decisional balance index and health-related quality of life (HRQoL) in APAP adherence. Furthermore, the results revealed an interaction between time and illness cognitive representations, and self-efficacy and family coping, in explaining adherence patterns over time. Therefore, understanding the causality of theoretically derived constructs is crucial to predict the continuity of APAP adherence.
\end{abstract}

Keywords: APAP treatment; adherence; illness cognitive representations; self-efficacy outcome expectations; family coping

\section{Introduction}

Adherence is established as one of the most common problems facing chronic diseases management. During five decades of research, concerted efforts have been made to understand adherence difficulties and identify predictors of non-adherence (DunbarJacob \& Mortimer-Stephens, 2001). Since its introduction, in 1981, by Sullivan, continuous positive airway pressure (CPAP) has become the widely accepted treatment of choice for patients with Obstructive Sleep Apnea (OSA), which requires consistent adherence. The recent alternative automatic positive airway pressure (APAP) device has prompted several studies to compare its effectiveness with CPAP (Galetke, Anduleit, Richter, Stieglitz, \& Randerath, 2008; Mulgrew, Cheema, Fleetham, Ryan, \& Ayas, 2007). As an example, our group recently showed the effectiveness of APAP therapy in controlling the majority of OSA symptoms beyond sleepiness and snoring (Cruz, Drummond, \& Winck, 2012). But, as in general, adherence results are mixed and the effect of APAP on enhancing adherence remains unclear (Haniffa, Lasserson, \& Smith, 2004). Although arbitrary, the used cut-off for no-adherence is based on minimal criteria for adequate sleep, this is less than $4 \mathrm{~h}$ for $70 \%$ of the treatment period (Collen, Lettieri,

*Corresponding author. Email: rute.s.sampaio@gmail.com 
Kelly, \& Roop, 2009; Engleman, Martin, \& Douglas, 1994; Stepnowsky \& Dimsdale, 2002).

Current relentless pursuit of predictors of adherence to CPAP has revealed mixed results in socio-demographic and clinical variables. Older age (Budhiraja et al., 2007; Woehrle, Graml, \& Weinreich, 2011) and being male (Joo \& Herdegen, 2007) are often related to adherence, although at odds (Lewis, Seale, Bartle, Watkins, \& Ebden, 2004). Even though the marital status does not predict adherence (Sucena, Liistro, Aubert, Rodenstein, \& Pieters, 2006), it seems to justify significant differences in a way of better adherence (Cartwright, 2008). An elevated basal (Apnoea Hypopnoea Index) AHI predicts adherence, at one and three months and CPAP treatment (Hui et al., 2001). Likewise, these results are not consistent (Gay, Weaver, Loube, \& Iber, 2006). These predictions failed to account for more than $10 \%$ of the variance in adherence (Collard, Pieters, Aubert, Delguste, \& Rodenstein, 1997). Despite the technological advances of CPAP equipment, their effect on adherence has not been steadily proved (Weaver \& Grunstein, 2008).

Regarding the multiplicity of psychological variables that have been studied (beliefs, self-efficacy, outcome expectations, social support, decisional balance, process of change and coping) the studies are unanimous in identifying these as main predictors of adherence, mostly based on theory-driven constructs (Aloia, Arnedt, Stanchina, \& Millman, 2007; Olsen, Smith, Oei, \& Douglas, 2008; Stepnowsky, Bardwell, Moore, Ancoli-Israel, \& Dimsdale, 2002; Stepnowsky \& Dimsdale, 2002; Stepnowsky, Marler, Palau, \& Annette Brooks, 2006; Tyrrell, Poulet, Pe Pin, \& Veale, 2006; Wild, Engleman, Douglas, \& Espie, 2004). Current approaches used to evaluate behavioral change are Bandura's Social cognitive theory (Bandura, 1997) (describing an agreement about the essential determinants of which health effective practices promote health) and Porchaska and DiClementes's Transtheoretical model (Prochaska, Redding, Harlow, Rossi, \& Velicer, 1994) (that focus the need for movement through discrete motivational stages over time and recognizing that individuals differ in their readiness for change) The advantage of psychological constructs is their potential targets for treatment that become enlarged (Aloia, Arnedt, Stepnowsky, Hecht, \& Borrelli, 2005). Psychological constructs are considered dynamic variables and self-efficacy has been pointed as a main predictor to implement efforts to persist in behavioral, when CPAP is already in use (Aloia et al., 2001). So, understanding how perceptions, experience and impact of having OSA might influence a patient's interpretation and response is still missing. Therefore Self-Regulatory Leventhal's model proposed an adaptive system, in which representation of illness experience (active parallel cognitive and emotional processing) might guide coping responses, followed by the appraisal of success/failure of those strategies (Leventhal, Leventhal, \& Cameron, 2001).

When facing a new diagnosis such as OSA and its treatment, family members are crucial. In fact, bed patterns are considered key promoters for the patient to seek help (McArdle, Kingshott, Engleman, Mackay, \& Douglas, 2001), as minimally symptomatic or asymptomatic OSA is estimated to occur in each of five subjects and often not recognized (Young, Peppard, \& Gottlieb, 2002). Although, the effect of family support and encouragement has not been tested in OSA, it makes intuitive sense important for adherence to APAP (Shapiro \& Shapiro, 2010). Thus, information regarding family coping, defined by a family's ability to cope with demands, family resources, and meanings associated with disease and subsequent implications (Montagnino \& Mauricio, 2004) could provide new information about what predicts adherence to APAP in a long term period. health-related quality of life (HRQoL) may function as a main outcome variable 
that embraces subjective patient's evaluation of impairment caused by treatment and the disease process impact (Gay et al., 2006; Joo \& Herdegen, 2007). Relationships between theoretically driven constructs related to adherence could therefore increase knowledge to design interventions to increase adherence to APAP.

Research, in the last decades, has explored several theory driven constructs to explain CPAP adherence, and although some identified variables play an important role, the relationship between these variables and over time remains unclear. According to previous studies, one pathway that needs to be more explored is the relationship among predictors of health behavior such as self-efficacy, outcome expectancies, illness and treatment perception and coping.

The present study has therefore focused on identifying the predictors of adherence to APAP treatment, during a six-month period, based on constructs derived from theoretical models focused on health-related behaviors. Also to address causality of its relationships was pointed as a main limitation by Aloia et al. (2005).

\section{Methods \\ Participants}

This is a prospective cohort study, with three assessments (baseline, T1, 1-2 months, T2 and 3-6 months, T3) conducted in a Sleep Disordered Breathing Clinic of a University Hospital in Northern Portugal. Between January 2009 and 2011, 153 consecutive OSA patients with APAP treatment indication underwent a standardized protocol (general clinic evaluation by sleep specialist, home sleep study, and psychological evaluation, including psychological morbidity, illness perceptions, self-efficacy, outcome expectations, social support, decision balance, change processes, family coping and quality of life). Exclusion criteria included the presence of psychiatric disorders or other sleep disorders. The study was approved by the hospital's research ethics committee and participants answered the written informed consent. Sixty-one patients were classified as poor adherent and 92 were classified as adherent at times two and three (Table 1).

\section{Disease assessment - home sleep study}

An overnight sleep study using a five-channel recording device (Sleep Screen, Viasys ${ }^{\text {TM }}$ Healthcare) was used, as previously described (Sampaio, Pereira, \& Winck, 2012), allowing objective measurement of APAP adherence. APAP was set with a minimum pressure of $4 \mathrm{~cm} \mathrm{H}_{2} \mathrm{O}$ and a maximum pressure of $15 \mathrm{~cm} \mathrm{H}_{2} \mathrm{O}$, by an experienced sleep physician.

Table 1. Cross-tabs descriptive analyses of adherence patterns in T2 and T3 $(n=153)$.

\begin{tabular}{llccr}
\hline & & \multicolumn{2}{c}{ Time 2 } \\
\cline { 3 - 5 } & & Poor adherents & Adherents & Total \\
\hline \multirow{2}{*}{ Time 3 } & Poor adherents & $50(82 \%)$ & $11(12 \%)$ & 61 \\
& Adherents & $11(18 \%)$ & $81(88 \%)$ & 92 \\
& Total & 61 & 92 & 153 \\
\hline
\end{tabular}




\section{Psychological assessment}

Initially a pilot study was conducted with all instruments and design procedures in 10 patients of the same hospital, with similar socio-demographic and clinical characteristics of the present sample. Upon CPAP prescription (T1), the following baseline questionnaires were administered, in face to face interview by a trained health psychologist and repeated in $\mathrm{T} 2$ and $\mathrm{T} 3$.

(1) Hospital Anxiety and Depression Scale (HADS) (Zigmond \& Snaith, 1983). HADS, is a 14-item scale with two subscales, measuring anxiety and depression, in a four point (0-3) Likert scale. Scores range from 0 to 21 , in both scales. A score above 11 (cut-off threshold) indicates a clinical diagnosis of anxiety and depression. In the current sample, internal consistency reliability coefficient (Cronbach, 1951) was adequate for both anxiety $(\alpha=.72)$ and depression subscales $(\alpha=.82)$, indicating good reliability.

(2) Determinants of nasal CPAP compliance (Stepnowsky, Marler, \& Ancoli-Israel, 2002). This instrument is based on two theories and has six scales: self-efficacy (four items), outcome expectations (four items), knowledge (11 items) and social support (nine items) based on socio cognitive theory (Bandura, 1997) and-decisional balance index (12 items) and process of change (20 items) based on a transtheoretical model (Prochaska et al., 1994). All scales require a (1-5) Likert response and higher scores indicate higher behavior engagement towards CPAP compliance. In the current sample, internal consistency reliability coefficient (Cronbach, 1951) was adequate for the following scales - self-efficacy $(\alpha=.87)$, outcome expectations $(\alpha=.75)$, social support $(\alpha=.94)$, decisional balance index $(\alpha=.86)$ and process of change $(\alpha=.88)$, indicating good reliability. Due to the fact that Cronbach alpha for the Knowledge scale $(\alpha=.53)$ was low it was not included in the hypothesis testing. The process of change subscale was completed only in T2 and T3 assessment moments, after CPAP's use.

(3) Illness Perception Questionnaire-Brief (Broadbent, Petrie, Main, \& Weinman, 2006). This instrument (Figueiras et al., 2010) has eight items, combined in three scales, that assesses illness perceptions: cognitive illness representations (consequences, timeline, personal control, treatment control, and identity); emotional representations (concern and emotions); and illness comprehensibility. All these items are rated in a ten-point Likert scale. This instrument also has another item, causal representation, which is answered in an open-ended response. High scores (sum of all items) indicated a more threatening perception of illness. In the current sample, internal consistency was between .60 and .72 .

(4) SAQLI Sleep Apnea Quality of Life Index (Sampaio et al., 2012) evaluates four domains of quality of life (daily functioning; social interactions; emotional functioning; symptoms) critical to this population and an additional domain (treatment related symptoms) evaluates treatment effect (used in T2 and T3). Scores are calculated as previously described (Sampaio et al., 2012) and higher scores indicated better quality of life. SAQLI presents a high internal consistency as reflected by Cronbach alpha coefficients from .84 to .89 (Sampaio et al., 2012). Pearson correlation coefficients between SAQLI interdomains before and after CPAP treatment and clinical variables were highly correlated (Sampaio et al., 2012).

(5) Family Crisis Oriented Evaluation Scales (F-Copes) (McCubbin, Larsen, \& Olson, 1982) measure how families respond/cope with problematic or difficult situations. Portuguese version includes five subscales: acquiring social support (six items); reframing (seven items); seeking spiritual support (three items); mobilizing the family to 
acquire and accept help (eight items); and passive appraisal (six items). Cronbach alpha for the total scale was .83. Responses were scored using a five-point likert scale (one, lower use of coping strategy and five, higher use of coping strategy). Higher scores indicate higher family coping strategies.

\section{Data analyses}

Descriptive analyses of socio-demographic variables (age, gender and marital status), clinical variables (AHI, mask leakage, $\mathrm{P} 95 \mathrm{~cm}$ percentile pressure-P95 which is the level of APAP pressure exceeded only $5 \%$ of the time, P95), and psychosocial variables (anxiety, depression, self-efficacy, outcome expectations, social support, decisional balance index, process of change, illness representations and family coping) were performed. Categorical variables were described as absolute frequencies and relative frequencies; mean and standard deviation were used for continuous variables.

The selected cut-off point for adherence was based on: the average number of hours used and the total usage percentage. So poorly adherent patients were those who used APAP $<4 \mathrm{~h} /$ night and $<70 \%$ total days of usage and adherent patients were those who used APAP $>4 \mathrm{~h} /$ night and $>70 \%$ total days of usage (Collen et al., 2009; Engleman et al., 1994; Stepnowsky \& Dimsdale, 2002).

To evaluate treatment adherence groups, multivariate models of generalized estimating equations (GEE) were used. GEE is a method to analyze longitudinal or repeated measures, taking into account that measurements of the same individual over time are correlated (Fitzmaurice, Laird, \& Ware, 2004). The advantage of this method is to provide consistent estimates of parameters associated with covariates in the model. A robust variance estimator was used to compute standard errors. Following the recommendations of Pepe \& Anderson (1994), we assume an exchangeable correlation matrix in the estimation of parameters of the models.

\section{Results}

Of the total 153 OSA patients, $61(40 \%)$ were poorly adherent and $92(60 \%)$ were adherent, at six months of APAP treatment. Poorly adherent patients used APAP on average $3: 12 \mathrm{~h}(\mathrm{SD}=1: 39 \mathrm{~h})$ and $3: 59(\mathrm{SD}=1: 50 \mathrm{~h})$ and 28 and $37 \%$ of days in Time 2 and 3, respectively. Adherent patients used APAP, on average, 6:28 h $(\mathrm{SD}=0: 59 \mathrm{~h})$ and $6.18 \mathrm{~h}(\mathrm{SD}=1: 15 \mathrm{~h})$ and 89 and $87 \%$ of days, in Time 2 and 3, respectively. The descriptive group analyses are presented in Table 1 at T2 and T3 of APAP adherence.

A multivariate model of GEE is presented in Table 3. Of the total variables pooled in the analysis, those who presented a significant univariate odds ratio like gender were included in the GEE models analysis as well as family coping because of its relation to CPAP adherence, in general. The descriptive statistics presented in Table 2 allowed for comprehensive results. Other psychological and clinical variables and its interactions were included in estimation models (processes of change, social support, illness emotional representation, anxiety, depression, mask leakage, AHI and P95). Notwithstanding, none of these variables revealed a significant interaction with regard to adherence pattern.

When adjusting for variables presented, it resulted in a model that includes age, HRQoL, self-efficacy, family coping and its interaction, decisional balance index and interaction between illness cognitive representations and time, that explained the adherence pattern over six months of treatment. 
Table 2. Socio-demographic, clinical, and psychological characteristics of poor adherents and adherents in T1-T3 $(n=153)$.

\begin{tabular}{|c|c|c|c|c|c|}
\hline & \multirow{2}{*}{$\begin{array}{c}\text { Time } 1 \\
\text { Total } n=153\end{array}$} & \multicolumn{2}{|c|}{ Time 2} & \multicolumn{2}{|c|}{ Time 3} \\
\hline & & $\begin{array}{c}\text { Poor adherents } \\
\quad n=61\end{array}$ & $\begin{array}{c}\text { Adherents } \\
n=92\end{array}$ & $\begin{array}{c}\text { Poor adherents } \\
\quad n=61\end{array}$ & $\begin{array}{c}\text { Adherents } \\
n=92\end{array}$ \\
\hline \multicolumn{6}{|c|}{ Socio-demographic variables } \\
\hline Gender & $\mathrm{n}(\%)$ & $\mathrm{n}(\%)$ & $\mathrm{n}(\%)$ & $\mathrm{n}(\%)$ & $\mathrm{n}(\%)$ \\
\hline Female & $28(18.3)$ & $10(6.54)$ & 18(11.76) & $12(7.84)$ & $16(10.46)$ \\
\hline \multirow[t]{2}{*}{ Male } & $125(81.7)$ & $51(33.33)$ & $74(48.37)$ & $49(32.03)$ & $76(49.67)$ \\
\hline & $\mathrm{M}(\mathrm{SD})$ & $\mathrm{M}(\mathrm{SD})$ & $\mathrm{M}(\mathrm{SD})$ & $\mathrm{M}(\mathrm{SD})$ & $\mathrm{M}(\mathrm{SD})$ \\
\hline Age & $52.2(10.3)$ & $50.9(10.79)$ & $53.1(9.86)$ & $49.5(9.68)$ & $54.1(10.28)$ \\
\hline \multicolumn{6}{|c|}{ Clinical variables } \\
\hline AHI & $43.0(19.0)$ & $4.7(3.79)$ & $4.7(5.21)$ & $4.5(4.38)$ & $4.3(5.33)$ \\
\hline $\begin{array}{l}\text { Mean leak } \\
\text { (L/min) }\end{array}$ & - & $26.9(14.56)$ & $29.6(17.01)$ & $24.8(17.41)$ & $27.6(14.17)$ \\
\hline $\begin{array}{l}\mathrm{P} 95 \\
\left(\mathrm{~cm} \mathrm{H} \mathrm{H}_{2} \mathrm{O}\right)\end{array}$ & - & $10.7(2.79)$ & $11.40(2.83)$ & $10.6(2.98)$ & $11.3(2.96)$ \\
\hline \multicolumn{6}{|c|}{ Determinates of nasal APAP } \\
\hline Self-efficacy & $4.5(1.17)$ & $3.8(.99)$ & $4.8(.47)$ & $3.9(1.2)$ & $4.7(.48)$ \\
\hline $\begin{array}{l}\text { Outcome } \\
\text { expectations }\end{array}$ & $4.3(.77)$ & $4.2(1.02)$ & $4.6(.57)$ & $4.2(.95)$ & $4.7(.61)$ \\
\hline $\begin{array}{l}\text { Social } \\
\text { support }\end{array}$ & $4.5(.85)$ & $4.1(1.18)$ & $4.5(.86)$ & $3.6(1.48)$ & $4.3(1.10)$ \\
\hline $\begin{array}{l}\text { Decisional } \\
\text { balance } \\
\text { index }\end{array}$ & $4.0(.57)$ & $3.6(.85)$ & $4.3(.60)$ & $3.6(.87)$ & $4.4(.06)$ \\
\hline $\begin{array}{l}\text { Processes of } \\
\text { change }\end{array}$ & - & $2.6(.97)$ & $2.7(.76)$ & $2.5(.85)$ & $2.6(.67)$ \\
\hline \multicolumn{6}{|l|}{$\begin{array}{c}\text { Psychological } \\
\text { variables }\end{array}$} \\
\hline Anxiety & $8.3(4.61)$ & $7.6(5.15)$ & $5.76(4.79)$ & $7.3(5.37)$ & $5.1(4.38)$ \\
\hline Depression & $5.7(3.66)$ & $4.8(4.13)$ & $3.8(3.47)$ & $4.4(4.43)$ & $3.4(3.6)$ \\
\hline $\begin{array}{l}\text { Illness } \\
\text { cognitive R. }\end{array}$ & $23.0(8.37)$ & $19.9(8.91)$ & $17.8(8.19)$ & $20.0(9.15)$ & $14.5(7.68)$ \\
\hline $\begin{array}{l}\text { Illness } \\
\text { emotional R. }\end{array}$ & $13.5(5.85)$ & $10.7(6.52)$ & $9.7(6.01)$ & $9.0(6.35)$ & $6.7(6.21)$ \\
\hline Family coping & $97.5(16.47)$ & $90.5(17.02)$ & 93.6(15.99) & $89.6(17.7)$ & $93.3(16.68)$ \\
\hline$H R Q o L$ & $4.5(1.28)$ & $4.9(1.35)$ & $5.7(.96)$ & $5.4(1.18)$ & $6.0(.77)$ \\
\hline
\end{tabular}

Notes: M - Mean; SD - Standard Deviation; AHI - Apnoea Hypopnoea Index; HRQL - Health-Related Quality of Life.

Among the socio-demographic variables, only age increased the probability of being adherent in 1.044 ( $p=.017$; OR: 1.042 ; IC95\%: $1.008-1.081)$. Gender is not a significant variable in this model, but from $\mathrm{T} 2$ to $\mathrm{T} 3$, female adherent percentage decreases and male adherence increased.

A unit increase in HRQoL was significantly related to adherence $(p=.006$; OR: 1.489 ; IC95\%: 1.122-1.977). In the first models tested, family coping was not associated with adherence $(p=.935$; OR: 1.002; IC95\%: .977-1.021), although self-efficacy increased its probability ( $p<.001$; OR: 2.047 ; IC95\%: 1.430-2.929). Interestingly, when these two variables were associated, odds of being adherent increased by 17 units $(p<.001$; OR: 17.443; IC95\%: 4.573-66.52). In the same way, family coping became significantly associated with adherence ( $p=.001$; OR: 1.107 ; IC95\%: 1.045-1.173). With a $5 \%$ error probability, this result showed a significant interaction between 
Table 3. GEE model for the adherence groups.

\begin{tabular}{|c|c|c|c|c|c|c|c|}
\hline & \multicolumn{7}{|c|}{ Adherence groups* } \\
\hline & $\mathrm{OR}(1)$ & CI95\% & & $P$ & $\mathrm{OR}(2)$ & CI95\% & \\
\hline \multicolumn{8}{|l|}{ Gender } \\
\hline Female** & .971 & .473 & 1.991 & .408 & 1.478 & .586 & 3.726 \\
\hline Male & 1.000 & - & - & - & 1.000 & - & - \\
\hline Age $^{1}$ & .968 & .939 & .997 & .017 & 1.044 & 1.008 & 1.081 \\
\hline HRQoL $^{1}-\mathrm{T} 1$ & 0983 & 0752 & 1286 & .549 & 1.076 & .774 & 1.618 \\
\hline HRQoL & 0649 & 0520 & 0810 & .006 & 1.489 & 1.122 & 1.977 \\
\hline Time & 1159 & 0878 & 1531 & - & - & - & - \\
\hline Self-efficacy $^{1}-\mathrm{T} 1$ & 1155 & 0930 & 1434 & .073 & .804 & .632 & 1.021 \\
\hline Self-efficacy & 0334 & 0238 & 0468 & .000 & 17.443 & 4.573 & 66.53 \\
\hline Time & 1073 & 0805 & 1432 & - & - & - & - \\
\hline Family coping ${ }^{1}-\mathrm{T} 1$ & 0999 & 0980 & 1018 & .950 & .999 & 0977 & 1022 \\
\hline Family coping & 0996 & 0981 & 1010 & .001 & 1.107 & 1.045 & 1.173 \\
\hline Time & 0998 & 0776 & 1282 & - & - & - & - \\
\hline Self-efficacy* family coping & - & - & - & .001 & .978 & .966 & .991 \\
\hline Illness cognitive $\mathrm{R}^{1}-\mathrm{T} 1$ & 1020 & 0983 & 1058 & .857 & .995 & .940 & 1.053 \\
\hline Illness cognitive $\mathrm{R}$ & 0960 & 0904 & 1020 & .008 & 1.115 & 1.029 & 1.209 \\
\hline Time & 0520 & 0282 & 0956 & - & - & - & - \\
\hline Illness cognitive $\mathrm{R}^{*}$ Time & 1039 & 1005 & 1073 & .033 & .951 & .908 & .996 \\
\hline Decisional balance index ${ }^{1}-\mathrm{T} 1$ & 1008 & 0958 & 1060 & .995 & 1.000 & .945 & 1.059 \\
\hline Decisional Balance index & 0907 & 0877 & 0939 & .024 & 1.050 & 1.006 & 1.095 \\
\hline Time & 1043 & 0787 & 1382 & - & - & - & - \\
\hline Time study & & & & .055 & 2.107 & .983 & 4.516 \\
\hline
\end{tabular}

Reference category: poor adherents;

**Females as reference category; OR(1) - Odds Ratio Univariate; OR(2) - Odds Ratio adjusted to all the variables in the model; CI95\% - Confidence interval; $p<.001 ; p<.05$.

${ }^{1}$ Continuous variable.

self-efficacy and family coping ( $p=.001$; OR: .978; IC95\%: .966-.991). So, when considering an increased self-efficacy and family coping, there is a slight probability of adherence increase. An increased unit in decisional balance index was also associated with adherence to APAP ( $p=.024$; OR: 1.050; IC95\%: 1.006-1.095).

Time had an effect on illness cognitive representations ( $p=.033$; OR: .951; IC95\%: .908-.996), presented in Figure 1. Association between time and threatening cognitive

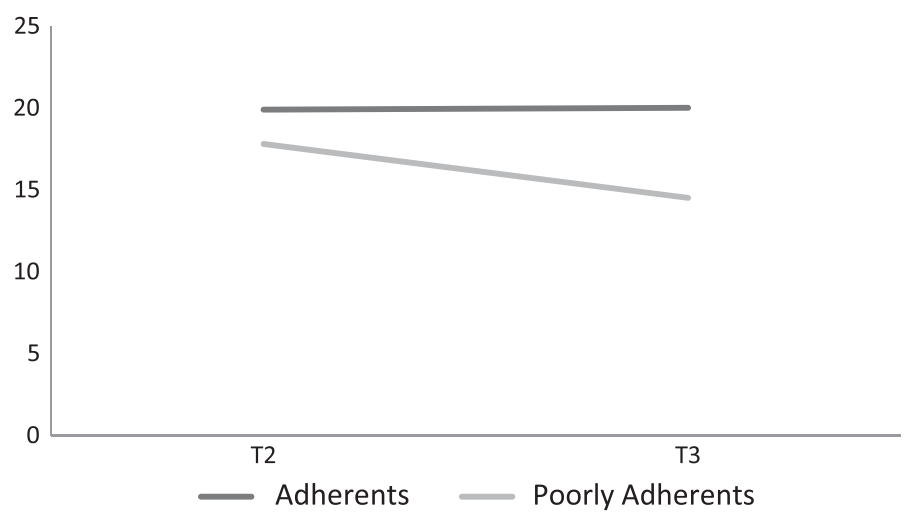

Figure 1. Time effect on illness cognitive representations. 
representations increased the probability of being poorly adherent, i.e. the adherent group perceived OSA as a less threatening disease (cognitive representations) over time.

\section{Discussion}

Results showed a high percentage of patients to be poorly adherent, even using APAP treatment, and a consistent pattern of adherence over time, according to the literature (Budhiraja et al., 2007; Weaver \& Sawyer, 2010). Like in previous studies referred (Aloia et al., 2007; Olsen et al., 2008; Stepnowsky, Bardwell, Moore, Ancoli-Israel, \& Dimsdale, 2002; Stepnowsky \& Dimsdale, 2002; Stepnowsky et al., 2006; Tyrrell et al., 2006; Wild et al., 2004), theoretically derived constructs only assumed a predicted value during treatment experience. Moreover, the nature of the relationship between behavior change and adherence increased with continued use of the treatment (Aloia et al., 2005). In fact, the literature is unanimous emphasizing the importance of including other psychological theoretical constructs, that might be useful in providing evidence for intervening in modifiable factors on APAP adherence (Aloia et al., 2005). Therefore, in the present study two important, and not yet described, theoretically derived constructs emerged as adherence predictors - illness cognitive representation and family coping. Illness cognitive representation is the only predictor of adherence that is dependent over time, i.e. as time passes, poor adherents perceive OSA and APAP treatment as slightly threatening. Conversely, in the adherent group, cognitive illness representations regarding OSA as a threatening illness decrease over time. This result is in accordance with the concept of self-regulation as a dynamic process, particularly in chronic diseases (Furze, Roebuck, Bull, Lewin, \& Thompson, 2002). This result that opens a window of intervention in the consistent early established adherence pattern may guide future interventions in order to reverse previous established consistence of adherence patterns.

Family coping, to our knowledge, has not been studied in OSA patients in need of APAP, a treatment also conspicuous for those who share the same living space. In one of the studies, previously presented (Stepnowsky et al., 2002), only active coping explained $16 \%$ of the variance in CPAP compliance in a set of measured variables. The present study emphasized the predictive role of family coping, regarding OSA and its treatment, when associated with self-efficacy. Self-efficacy has been known to be an important predictor of adherence (Aloia et al., 2007; Olsen et al., 2008; Stepnowsky, Bardwell, Moore, Ancoli-Israel, \& Dimsdale, 2002), but clearly, in our sample, higher family coping strategies seem to reinforce the way that the patients perceive themselves to be capable to respond to APAP treatment, in a wholeness interrelation. Another important psychological construct emerged, the decisional balance index, where the pros to continue to adhere to APAP treatment reinforce its usage. At least, as expected and widely documented, the key clinical factor pointed about the cost-effectiveness of treatment of OSA is HRQoL improvement (Mar, Rueda, Duran-Cantolla, Schechter, \& Chilcott, 2003), which may reinforce continuing APAP usage and indeed predict adherence. Our study also reinforces previous results of older age as a predictor of adherence (Budhiraja et al., 2007). Although gender did not predict adherence, our results are consistent with other studies (Joo \& Herdegen, 2007), and there is a slight decrease in APAP usage by women, over time, contrary to male patients.

Several limitations in the present study need to be acknowledged. The first concerns the generalizations of results due to the single country nature of this study. Like other longitudinal studies, $25 \%$ of patients were lost between T1 and T3. A second limitation 
that needs to be addressed is the defined timelines chosen for practical reasons i.e. when patients had to come for their appointments (outpatient visit) to the Sleep Disordered Breathing Clinic. Another caveat is, due to the prevalence of OSA, that the sample reflects an imbalanced distribution representing the normal ratio between female and male OSA patients. Not considering subjective sleepiness in the protocol can be other limitation. Finally, although there is no precise key number for GEE analysis, it is recognized that the bigger the sample, the lower the amplitude of CI.

The present study shows important strengths, particularly the importance of theoretically derived constructs regarding adherence to APAP and the application of GEE's statistical models, over time.

The results of this study guide prediction models related to adherence on OSA patients. Further studies should focus on family variables like family coping and cognitive representations regarding OSA, in APAP adherence, in periods over six months, particularly in poorly adherent patients.

\section{Conclusion and clinical implications}

In conclusion, the results showed the importance of theoretically derived constructs in predicting APAP adherence. In fact, APAP may provide better control of OSA increasing patients' outcome expectancies regarding APAP treatment. At the same time, with APAP, patients may perceive themselves as self-efficient in managing the disease taking in to consideration family coping strategies that may function as cues to action feeding self-efficacy, as well and as a result, improving their quality of life.

Taking the results in to consideration, it is important for health professionals to assess patients and differentiate adherence patterns, in order to individualize interventions tailored to the patients' adherence patterns. Interventions, during APAP treatment, that take in to consideration the patient's age and include a key family member like a partner are crucial in promoting adherence to APAP. As a result, intervention in OSA and particularly in APAP adherence should be focused on the family as the unit of treatment.

\section{Conflict of interest}

None of the authors have any financial or other relationships that might lead to conflict of interest.

\section{Acknowledgments}

This work was supported by a grant (SFRH/BD/38388/2007) from the Portuguese Foundation of Science and Technology. We thank all patients that agreed to participate in this study.

\section{References}

Aloia, M.S., Arnedt, J.T., Smith, L., Skrekas, J., Stanchina, M., \& Millman, R.P. (2005). Examining the construct of depression in obstructive sleep apnea syndrome. Sleep Medicine, 6, 115-121. doi: 10.1016/j.sleep.2004.09.003

Aloia, M.S., Arnedt, J.T., Stanchina, M., \& Millman, R.P. (2007). How early in treatment is PAP adherence established? Revisiting night-to-night variability. Behavioral Sleep Medicine, 5, 229-240. doi: 10.1080/15402000701264005 
Aloia, M.S., Arnedt, J.T., Stepnowsky, C., Hecht, J., \& Borrelli, B. (2005). Predicting treatment adherence in obstructive sleep apnea using principles of behavior change. Journal of Clinical Sleep Medicine, 1, 346-353.

Aloia, M.S., Di Dio, L., Ilniczky, N., Perlis, M.L., Greenblatt, D.W., \& Giles, D.E. (2001). Improving compliance with nasal CPAP and vigilance in older adults with OAHS. Sleep Breath, 5, 13-21. doi: 10.1007/s11325-001-0013-9

Bandura, A. (1997). Self-efficacy: The exercise of control. New York, NY: W.H. Freeman.

Broadbent, E., Petrie, K.J., Main, J., \& Weinman, J. (2006). The brief illness perception questionnaire. Journal of Psychosomatic Research, 60, 631-637. doi: 10.1016/j.jpsychores.2005.10.020

Budhiraja, R., Parthasarathy, S., Drake, C.L., Roth, T., Sharief, I., Budhiraja, P., ... Hudgel, D.W. (2007). Early CPAP use identifies subsequent adherence to CPAP therapy. Sleep, 30, 320-324.

Cartwright, R. (2008). Sleeping together: A pilot study of the effects of shared sleeping on adherence to CPAP treatment in obstructive sleep apnea. Journal of Clinical Sleep Medicine, 4, 123-127.

Collard, P., Pieters, T., Aubert, G., Delguste, P., \& Rodenstein, D.O. (1997). Compliance with nasal CPAP in obstructive sleep apnea patients. Sleep Medicine Reviews, 1, 33-44.

Collen, J., Lettieri, C., Kelly, W., \& Roop, S. (2009). Clinical and polysomnographic predictors of short-term continuous positive airway pressure compliance. Chest, 135, 704-709. doi: $10.1378 /$ chest. $08-2182$

Cronbach, L.J. (1951). Coefficient alpha and the internal structure of tests. Psychometrika, 16, 297-334.

Cruz, I.A., Drummond, M., \& Winck, J.C. (2012). Obstructive sleep apnea symptoms beyond sleepiness and snoring: Effects of nasal APAP therapy. Sleep Breath, 16, 361-366. doi: $10.1007 / \mathrm{s} 11325-011-0502-4$

Dunbar-Jacob, J., \& Mortimer-Stephens, M.K. (2001). Treatment adherence in chronic disease. Journal of Clinical Epidemiology, 54, S57-60.

Engleman, H.M., Martin, S.E., \& Douglas, N.J. (1994). Compliance with CPAP therapy in patients with the sleep apnoea/hypopnoea syndrome. Thorax, 49, 263-266.

Figueiras, M., Marcelino, D.S., Claudino, A., Cortes, M.A., Maroco, J., \& Weinman, J. (2010). Patients' illness schemata of hypertension: The role of beliefs for the choice of treatment. Psychology \& Health, 25, 507-517. doi: 10.1080/08870440802578961

Fitzmaurice, G.M., Laird, N.M., \& Ware, J.H. (2004). Applied Longitudinal Analysis. Hoboken, NJ: Wiley-Interscience.

Furze, G., Roebuck, A., Bull, P., Lewin, R.J., \& Thompson, D.R. (2002). A comparison of the illness beliefs of people with angina and their peers: A questionnaire study. BMC Cardiovascular Disorders, 2, 1-5.

Galetke, W., Anduleit, N., Richter, K., Stieglitz, S., \& Randerath, W.J. (2008). Comparison of automatic and continuous positive airway pressure in a night-by-night analysis: A randomized, crossover study. Respiration, 75, 163-169. doi: 10.1159/000097767

Gay, P., Weaver, T., Loube, D., \& Iber, C. (2006). Evaluation of positive airway pressure treatment for sleep related breathing disorders in adults. Sleep, 29, 381-401.

Haniffa, M., Lasserson, T.J., \& Smith, I. (2004). Interventions to improve compliance with continuous positive airway pressure for obstructive sleep apnoea. Cochrane Database of Systematic Reviews, CD003531. doi:10.1002/14651858.CD003531.pub2

Hui, D.S., Choy, D.K., Li, T.S., Ko, F.W., Wong, K.K., Chan, J.K., \& Lai, C.K. (2001). Determinants of continuous positive airway pressure compliance in a group of Chinese patients with obstructive sleep apnea. Chest, 120, 170-176.

Joo, M.J., \& Herdegen, J.J. (2007). Sleep apnea in an urban public hospital: Assessment of severity and treatment adherence. Journal of Clinical Sleep Medicine, 3, 285-288.

Leventhal, H., Leventhal, E., \& Cameron, L. (2001). Representations, procedures, and affect in illness self-regulation: A perceptual-cognitive model. In A. Baum, T. Revenson, \& J. Singer (Eds.), Handbook of health psychology (pp. 19-48). Abingdon: Lawrence Erlbaum associates.

Lewis, K.E., Seale, L., Bartle, I.E., Watkins, A.J., \& Ebden, P. (2004). Early predictors of CPAP use for the treatment of obstructive sleep apnea. Sleep, 27, 134-138.

Mar, J., Rueda, J.R., Duran-Cantolla, J., Schechter, C., \& Chilcott, J. (2003). The cost-effectiveness of nCPAP treatment in patients with moderate-to-severe obstructive sleep apnoea. European Respiratory Journal, 21, 515-522. 
McArdle, N., Kingshott, R., Engleman, H.M., Mackay, T.W., \& Douglas, N.J. (2001). Partners of patients with sleep apnoea/hypopnoea syndrome: Effect of CPAP treatment on sleep quality and quality of life. Thorax, 56, 513-518.

McCubbin, H.I., Larsen, A., \& Olson, D.H. (1982). Family crisis oriented personal evaluation scales. In D.H. Olson, H.I. McCubbin, H. Barnes, A. Larsen, M. Muxen, \& M. Wilson (Eds.), Family inventories (pp. 143-159). St. Paul, MN: University of Minnesota Press.

Montagnino, B.A., \& Mauricio, R.V. (2004). The child with a tracheostomy and gastrostomy: Parental stress and coping in the home-a pilot study. Pediatric Nursing, 30(5), 373-380, 401.

Mulgrew, A.T., Cheema, R., Fleetham, J., Ryan, C.F., \& Ayas, N.T. (2007). Efficacy and patient satisfaction with autoadjusting CPAP with variable expiratory pressure vs. standard CPAP: A two-night randomized crossover trial. Sleep Breath, 11, 31-37. doi: 10.1007/s11325-0060078-6

Olsen, S., Smith, S., Oei, T., \& Douglas, J. (2008). Health belief model predicts adherence to CPAP before experience with CPAP. European Respiratory Journal, 32, 710-717. doi: $10.1183 / 09031936.00127507$

Pepe, M.S., Anderson, G.L. (1994). A cautionary note on inference for marginal regression models with longitudinal data and general correlated response data. Communications in StatisticsSimulation, 23, 939-951.

Prochaska, J.O., Redding, C.A., Harlow, L.L., Rossi, J.S., \& Velicer, W.F. (1994). The transtheoretical model of change and HIV prevention: A review. Health Education Quarterly, 21, 471-486.

Sampaio, R.S., Pereira, M.G., \& Winck, J.C. (2012). Adaptation of the sleep apnea quality of life index (SAQLI) to portuguese obstructive sleep apnea syndrome patients. Revista Portuguesa Pneumologia. doi:10.1016/j.rppneu.2012.02.009

Shapiro, G., \& Shapiro, C. (2010). Factors that influence CPAP adherence. An overview. Sleep and Breathing, 14, 323-335. doi: 10.1007/s11325-010-0391-y

Stepnowsky, C.J.Jr., Bardwell, W.A., Moore, P.J., Ancoli-Israel, S., \& Dimsdale, J.E. (2002). Psychologic correlates of compliance with continuous positive airway pressure. Sleep, 25, 758-762.

Stepnowsky, C., \& Dimsdale, J. (2002). Dose-response relationship between CPAP compliance and measures of sleep apnea severity. Sleep Medicine, 3, 329-334.

Stepnowsky, C.J.Jr., Marler, M.R., \& Ancoli-Israel, S. (2002). Determinants of nasal CPAP compliance. Sleep Medicine, 3, 239-247.

Stepnowsky, C.J., Marler, M.R., Palau, J., \& Annette Brooks, J. (2006). Social-cognitive correlates of CPAP adherence in experienced users. Sleep Medicine, 7, 350-356. doi: 10.1016/j. sleep. 2005.11.004

Sucena, M., Liistro, G., Aubert, G., Rodenstein, D.O., \& Pieters, T. (2006). Continuous positive airway pressure treatment for sleep apnoea: Compliance increases with time in continuing users. European Respiratory Journal, 27, 761-766. doi: 10.1183/09031936.06.00087705

Tyrrell, J., Poulet, C., Pe Pin, J.L., \& Veale, D. (2006). A preliminary study of psychological factors affecting patients' acceptance of CPAP therapy for sleep apnoea syndrome. Sleep Medicine, 7, 375-379. doi: 10.1016/j.sleep. 2005.10.005

Weaver, T.E., \& Grunstein, R.R. (2008). Adherence to continuous positive airway pressure therapy: The challenge to effective treatment. Proceedings of American Thoracic Society, 5, 173-178. doi: $10.1513 /$ pats.200708-119MG

Weaver, T.E., \& Sawyer, A.M. (2010). Adherence to continuous positive airway pressure treatment for obstructive sleep apnoea: Implications for future interventions. The Indian Journal of Medical Research, 131, 245-258.

Wild, M.R., Engleman, H.M., Douglas, N.J., \& Espie, C.A. (2004). Can psychological factors help us to determine adherence to CPAP? A prospective study European Respiratory Journal, 24, 461-465. doi: 10.1183/09031936.04.00114603

Woehrle, H., Graml, A., \& Weinreich, G. (2011). Age- and gender-dependent adherence with continuous positive airway pressure therapy. Sleep Medicine, 12, 1034-1036.

Young, T., Peppard, P.E., \& Gottlieb, D.J. (2002). Epidemiology of obstructive sleep apnea: A population health perspective. American Journal of Respiratory and Critical Care Medicine, $165,1217-1239$.

Zigmond, A.S., \& Snaith, R.P. (1983). The hospital anxiety and depression scale. Acta Psychiatrica Scandinavica, 67, 361-370. 